\title{
Development and Verification of Cooperative Adaptive Cruise Control via LTE- V
}

\author{
Ping Wang ${ }^{1}$, Yuqi Chen ${ }^{1}$, Chao Wang ${ }^{2}$, Fuqiang $\mathrm{Liu}^{1}$, Jia $\mathrm{Hu}^{3 *}$, and Nguyen Ngoc Van ${ }^{4}$ \\ ${ }^{1}$ Department of Information and Communication Engineering, Tongji University, Shanghai, China \\ ${ }^{2}$ Department of Computer Science, University of Exeter, Exeter, U.K. \\ ${ }^{3}$ Department of Transport Engineering, Tongji University, Shanghai, China \\ ${ }^{4}$ School of Electronics and Telecommunications, Hanoi University of Science and Technology, Hanoi, Vietnam \\ "hujia@tongji.edu.cn
}

\begin{abstract}
In this paper, we present a testbed platform for realizing cooperative adaptive cruise control (CACC) enabled by LTE-V (LTE-vehicle). The platform is developed on a platoon of vehicles, each of which is equipped with a suite of on-board sensing and computing devices for environment perception and automated vehicle control, as well as an LTE-V transceiver for high-performance vehicle-to-vehicle (V2V) communication. The hardware architecture and software architecture, especially the perception and control methods, of the platform are described. Field experiments in different road conditions are conducted to verify the feasibility of our platform. The results also show the potential of V2V communications via LTE-V in terms of improving the sensing capability of individual vehicle's on-board sensors.
\end{abstract}

\section{Introduction}

The demand for road transportation is increasing at a rapid pace in most countries around the world. This causes serious road safety and traffic congestion problems, and also leads to huge fuel consumption and environmental pollution issues. Based on the concept of cooperative automated driving [1], grouping vehicles on the road into platoons is one possible technical solution to these problems. Fig. 1 illustrates a traffic scene which contains a platoon formed of three vehicles. The leading vehicle (vehicle No. 1) can be driven by human. If the first following vehicle (vehicle No. 2) and the second following vehicle (vehicle No. 3) are capable of autonomously adjusting their speed in order to keep a constant distance from their respective front vehicles, road safety can be improved by replacing human drivers with intelligent automated driving functions. Higher traffic efficiency [2-7] is achievable because more road space can be saved for other vehicles. Fuel consumption [8-12] can also be decreased due to the improved coordinated driving actions and shorter inter-vehicle distance which reduces air resistance. This helps relieve environment pollution [13].

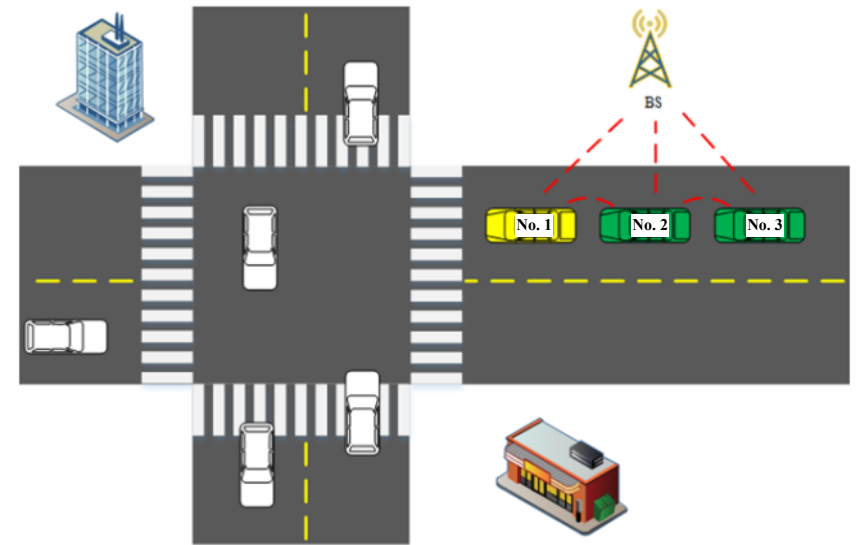

Fig. 1 A platoon of vehicles on the road.
There have been a good number of research projects [14] working on cooperative automated driving. For instance, the California Partners for Advanced Transportation Technology (PATH) project focused on heavy trucks and its experiments demonstrated the technical feasibility of platooning three trucks at the speed of $105 \mathrm{~km} / \mathrm{h}$ with an intervehicle gap of only $18 \mathrm{~m}$ [15] [16]. The Energy ITS project [17] was funded by the Japanese Ministry of Economy, Trade and Industry. It aimed at achieving energy saving and global warming prevention with intelligent transportation system (ITS) technologies. An expressway experiment was conducted which formed an automated truck platoon using three heavy trucks and one light truck, with the inter-vehicle gaps of $10 \mathrm{~m}$ and $4.7 \mathrm{~m}$ respectively at the speed of $80 \mathrm{~km} / \mathrm{h}$.

For each following vehicle, the most important functions for realizing the concept of cooperative adaptive cruise control (CACC) [18] are accurately detecting the position and status of its front vehicle (termed perception), and making correct decision for future action and then carrying out the plan in order to safely and smoothly follow the front vehicle (termed control). Most existing perception and control solutions heavily rely on the sensing data collected through various on-board sensors such as cameras and radars [19-22]. For example, in [23] a camera (MobilEye) mounted on the front windshield is used for lane and object detection. In [24] multiple cameras including a frontview stereo camera and several sideview cameras are utilized for object detection, and a colour camera is used for traffic light detection. However, the sensing range and accuracy of sensors are in general limited, especially in complex traffic conditions. Hence, the knowledge regarding the cooperative vehicles within the platoon and other objects (such as vehicles, pedestrians, bicycles, and so forth) outside the platoon that can be attained by each vehicle may not be fully satisfactory when the platoon speed is high and the inter-vehicle distance is small.

To handle this issue, the vehicle-to-everything (V2X) communication technology, which allows vehicles to collaborate with each other by exchanging real-time state 
information (e.g., speed, wheel angle, heading and location, throttle and brake actions, etc.) to achieve better environment perception, has attracted wide research attentions. The dedicated short-range communications (DSRC) technology has been considered as the solution to realizing V2X communication for years. The US Federal Communication Commission (FCC) has allocated a $75 \mathrm{MHz}$ spectrum in the $5.9 \mathrm{GHz}$ band for DSRC [25]. The information exchanged among vehicles can be encoded into Basic Safety Message (BSM) and periodically broadcasted by vehicles according to the Society of Automotive Engineering (SAE) standard [26]. However, due to the ad hoc working nature, DSRC may not be able to fully guarantee highly-reliable and low-delay communication in all traffic conditions.

In the past few years, the cellular-based LTE-V (LTE vehicle) technology [27] has started to be investigated in many countries and research communities. For example, a data exchange standard [28] is currently being developed by the Society of Automotive Engineers of China (SAE-China). Cooperative automated driving is seen as one of the most important use cases supported by LTE-V and LTE-eV2X (the next generation of LTE-V) according to 3GPP TR 22.886 [29]. LTE-V supports both V2V (vehicle-to-vehicle) communication over the peer-to-peer PC5 interface, and V2N (vehicle-to-network) communication over the LTE-Uu interface. Therefore, the interference management and scheduling of $\mathrm{V} 2 \mathrm{~V}$ communication traffic can be assisted by base stations to obtain a more reliable and efficient message dissemination compared with DSRC, especially in the highly mobile environment. The transmission delay of LTE-V is expected to be about $25 \mathrm{~ms}$ through $\mathrm{V} 2 \mathrm{~V}$ direct communication, which meets the latency requirements of platooning defined in 3GPP TR 22.886. A comparative analysis between LTE-V and DSRC [30] is shown in Table 1, which clearly demonstrates the advantages of the former technology.

Table 1 LTE-V vs DSRC [30]

\begin{tabular}{|c|c|c|c|}
\hline & LTE-V & DSRC & $\begin{array}{l}\text { Advantages } \\
\text { of LTE-V }\end{array}$ \\
\hline $\begin{array}{l}\text { Synch- } \\
\text { ronous } \\
\text { mode }\end{array}$ & Synchronous & Asynchronous & $\begin{array}{l}\text { High spectral } \\
\text { efficiency }\end{array}$ \\
\hline $\begin{array}{l}\text { Resource } \\
\text { reuse }\end{array}$ & $\begin{array}{l}\text { FDM and } \\
\text { TDM }\end{array}$ & TDM & $\begin{array}{l}\text { Sensitivity } \\
\text { gain and large } \\
\text { link budgets }\end{array}$ \\
\hline $\begin{array}{l}\text { Channel } \\
\text { estimation }\end{array}$ & $\begin{array}{l}\text { Four } \\
\text { symbol/frame }\end{array}$ & $\begin{array}{l}\text { One } \\
\text { symbol/frame }\end{array}$ & $\begin{array}{l}\text { Accurate and } \\
\text { reliable }\end{array}$ \\
\hline $\begin{array}{l}\text { Channel } \\
\text { coding }\end{array}$ & Turbo & $\begin{array}{l}\text { Convolutional } \\
\text { code }\end{array}$ & $\begin{array}{l}\text { Low error } \\
\text { rate }\end{array}$ \\
\hline $\begin{array}{l}\text { Retrans- } \\
\text { mission }\end{array}$ & HARQ & No HARQ & $\begin{array}{l}\text { Reliable } \\
\text { performance }\end{array}$ \\
\hline Modulation & SC-FDM & OFDM & $\begin{array}{l}\text { High power } \\
\text { efficiency }\end{array}$ \\
\hline
\end{tabular}

Although the potentials of CACC facilitated by LTE$\mathrm{V}$ have been warmly discussed, the major part of current research works are theoretic and/or simulation-based. System designs from the practical viewpoints and field experiments are relatively limited. In this paper, we aim to present our solution. Specifically, we develop a testbed platform for realizing $\mathrm{CACC}$ via a number of vehicles, with a software system that can realize perception and control functions. The hardware of the platform is formed of vehicles equipped with a suite of on-board sensing devices (e.g., in-vehicle kinetic sensors, camera, radar, DGPS, etc.) and high-performance computing units to perform environment sensing, and with an LTE-V transceiver to realize real-time V2V information exchange. The software architecture consists of five layers, through which each following vehicle conducts accurate measurements of the position and status of the cooperative vehicles within the platoon and also possibly other noncooperative objects (vehicles, bicycles, pedestrians, obstacles, etc.) in the surrounding driving environment, and then carries out vehicle control in order to autonomously follow the trajectory of the leading vehicle while maintaining a safe and short distance to the front vehicle.

Good perception and control algorithms can contribute to reliability and stability of the platoon. Many existing autonomous driving and adaptive cruise control (ACC) applications use only on-board sensors to support the perception function. In our system the measurements attained from sensors and $\mathrm{V} 2 \mathrm{~V}$ communication are fused to attain a better representation of the environment. In addition, based on the model predictive control (MPC) framework [31-34], a temporal longitudinal controller and a spatial lateral controller are developed together for smooth and safe vehicle manoeuvring. A number of field experiments are carried out to evaluate the performance of the perception and control methods of our platform in different road conditions. The results verify the feasibility and functioning of our system, as well as the potential of $\mathrm{V} 2 \mathrm{~V}$ communications via LTE-V in terms of improving the sensing capability of individual vehicle's on-board sensors.

The remainder of the paper is organized as follows. In Section 2 and Section 3, the hardware and software architectures of our platform are described, respectively. Details of the perception algorithm are presented in Section 4. In Section 5, the low-level control algorithm is explained. Experimental scenarios and results are discussed in Section 6. Finally, Section 7 concludes the paper.

\section{Hardware Architecture}

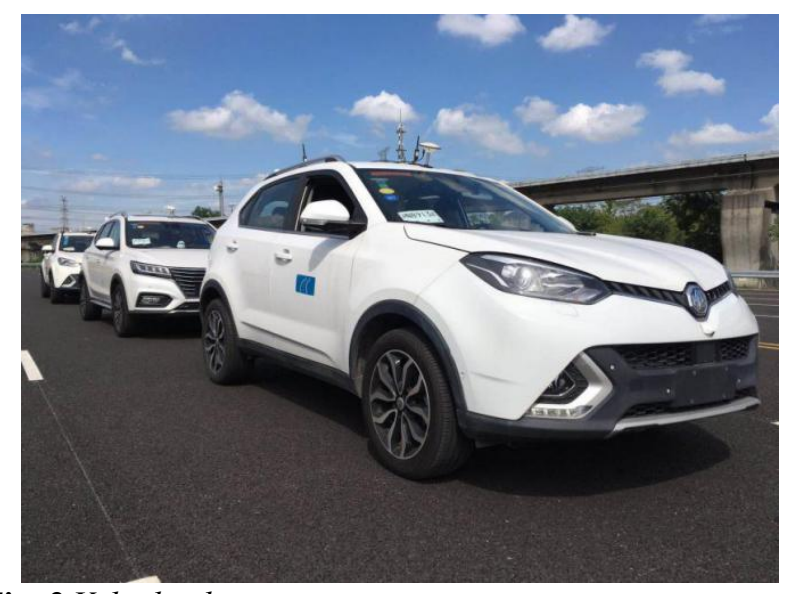

Fig. 2 Vehicle platoon

Three SAIC Morris Garages cars are used to form our testbed, as shown in Fig. 2. Fig. 3 illustrates the schematic overview of the hardware architecture. In addition to invehicle kinetic sensors, each vehicle is equipped with a suite of advanced on-board sensors, including a DGPS (differential 
global positioning system) receiver, a front-facing millimeter-wave radar, and a camera. All these devices are connected to the host vehicle's gateway via the Controller Area Network (CAN). The gateway enables all foundational interactions among different devices. It provides the CACC software platform, operated on an electronic computing unit (NVIDIA Drive PX2), with necessary sensing data, and also converts control signals (e.g., desired acceleration and desired future trajectory) output from the platform into appropriate action signals to manoeuvre the vehicle. In our system, the gateway allows a human driver to take over the vehicle from the automated driving operation, by pressing brake pedal or turning steering wheel. The hardware platform also contains an LTE-V transceiver (a prototype for V2V communication enabled by Huawei Balong765 [27]) and an HMI device (Huawei M2-A01L tablet). The HMI displays all status information regarding the platoon to the human driver. It can also be used by the driver to send orders to join or leave a platoon.
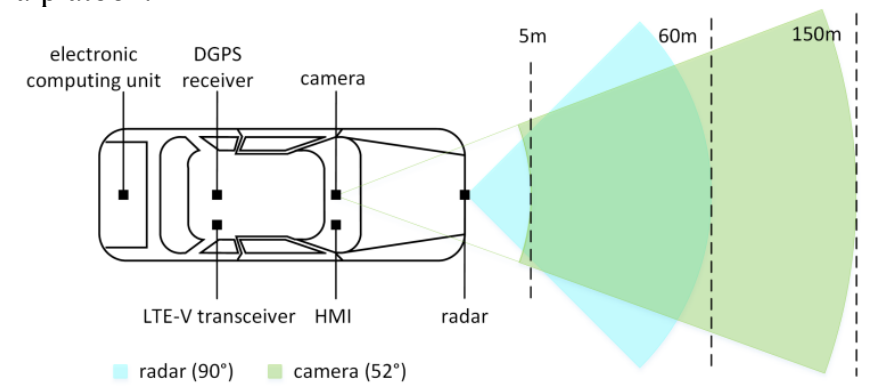

Fig. 3 Schematic overview of hardware architecture.

The radar (Delphi ESR) is mounted on the host vehicle's front bumper. It can simultaneously detect up to 64 objects, with a maximum detection range of $60 \mathrm{~m}$ and a horizontal detection angle of 90 degrees. The camera (Mobileye) is mounted on the front windshield. Its detection algorithm has a maximum detection range of $150 \mathrm{~m}$ and minimum detection distance of $5 \mathrm{~m}$, and can detect up to 10 objects at the same time. The DGPS receiver provides the host vehicle with precise positioning information including longitude, latitude, heading and velocity. The accuracy of the location is normally within $0.01 \mathrm{~m}$ and that of the heading is within 1 degree.

\section{Software Architecture}

Following [35], a general software architecture of a cooperative automated driving system consists of three parts: model data supplier, model data processor and model data consumer. A model data supplier is responsible for collecting data from various on-board devices. A model data processor processes the collected data to satisfy different functional requirements. The results are then sent to the model data consumer for driving system controlling. Inspired by this architecture, five layers are developed in our platform to support CACC as shown in Fig. 4. An input layer conducts the function of the model data supplier and provides input data to the platform. A perception layer, a world model layer and a low-level control layer represent the model data processor, where the perception layer aims to detect surrounding objects, the world model layer collects and handles the data from the perception layer to generate a world model, and the low-level control layer calculates appropriate action signals. Finally, an output layer can be considered as the model data consumer. It feeds the results generated by the platform to other devices, for control, display, and/or V2X transmission. The detailed explanations of each layer are presented as follows.

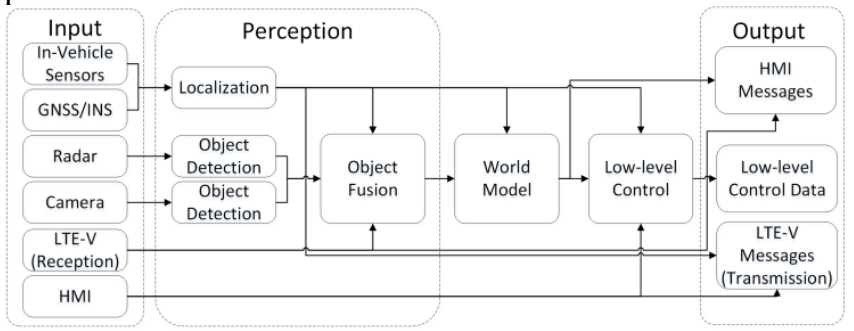

Fig. 4 Schematic overview of software architecture.

\subsection{Input layer}

All the necessary input data useful for perception of driving environment and control of vehicle are collected in this layer. The input data include the kinetic state data of the host vehicle measured by in-vehicle sensors, sensing data collected from on-board radar and camera, positioning information of the host vehicle attained from the DGPS receiver, $\mathrm{V} 2 \mathrm{~V}$ messages received from LTE-V transceiver, and control messages input from HMI by the driver. The details of these data are displayed in Table 2.

Table 2 Input layer data

\begin{tabular}{|c|c|c|c|}
\hline $\begin{array}{l}\text { Data } \\
\text { source }\end{array}$ & $\begin{array}{l}\text { Collection } \\
\text { Frequency }\end{array}$ & $\begin{array}{c}\text { Data } \\
\text { description }\end{array}$ & Data contents \\
\hline $\begin{array}{l}\text { Gateway } \\
\text { (kinetic } \\
\text { sensors) }\end{array}$ & $100 \mathrm{~Hz}$ & $\begin{array}{l}\text { Host vehicle } \\
\text { state }\end{array}$ & $\begin{array}{l}\text { Velocity }(\mathrm{m} / \mathrm{s}) \\
\text { Acceleration }\left(\mathrm{m} / \mathrm{s}^{2}\right) \\
\text { Wheel angle } \\
\text { (degree) }\end{array}$ \\
\hline Radar & $20 \mathrm{~Hz}$ & $\begin{array}{l}\text { Radar } \\
\text { measurements }\end{array}$ & $\begin{array}{l}\text { Position }(\mathrm{m}) \\
\text { Velocity }(\mathrm{m} / \mathrm{s})\end{array}$ \\
\hline Camera & $20 \mathrm{~Hz}$ & $\begin{array}{l}\text { Camera } \\
\text { measurements }\end{array}$ & $\begin{array}{l}\text { Position }(\mathrm{m}) \\
\text { Velocity }(\mathrm{m} / \mathrm{s})\end{array}$ \\
\hline $\begin{array}{l}\text { DGPS } \\
\text { receiver }\end{array}$ & $50 \mathrm{~Hz}$ & $\begin{array}{l}\text { Global } \\
\text { positioning } \\
\text { state }\end{array}$ & $\begin{array}{l}\text { Longitude (degree) } \\
\text { Latitude (degree) } \\
\text { Heading (degree) }\end{array}$ \\
\hline HMI & $20 \mathrm{~Hz}$ & $\begin{array}{l}\text { Platooning } \\
\text { control } \\
\text { messages }\end{array}$ & $\begin{array}{l}\text { Joining, leaving, } \\
\text { establishing, or } \\
\text { dissolving platoon }\end{array}$ \\
\hline \multirow[t]{2}{*}{$\begin{array}{l}\text { LTE-V } \\
\text { trans- } \\
\text { ceiver }\end{array}$} & $50 \mathrm{~Hz}$ & $\begin{array}{l}\text { Other vehicle } \\
\text { state }\end{array}$ & $\begin{array}{l}\text { Vehicle identity } \\
\text { Vehicle state } \\
\text { Global positioning } \\
\text { state }\end{array}$ \\
\hline & & $\begin{array}{l}\text { Platoon } \\
\text { broadcast } \\
\text { information }\end{array}$ & $\begin{array}{l}\text { Platoon state } \\
\text { Platoon control }\end{array}$ \\
\hline
\end{tabular}

\subsection{Perception layer}

The perception layer first tracks the objects detected by different sensors (radar, camera, and LTE-V transceiver) and then fuses all the tracks to provide a unified understanding of objects in the driving environment. The perception process is divided into several steps, including coordinate transformation, multi-object tracking, track-totrack association, and data fusion. The whole process is executed iteratively with a fixed frequency of $50 \mathrm{~Hz}$. At the beginning of each iteration, new measurements of the positioning information of the front vehicle, leading vehicle, 
and possibly other ambient objects are attained by radar, camera, and LTE-V transceiver. Since different sensors collect data in different forms and coordinate systems, a coordinate transformation step is first taken to unify the coordinate system. The multi-object tracking step aims to match new measurements of each sensor to its previously tracked objects in earlier iterations, in order to update the knowledge of the objects' status. Such a task is conducted using each data source (i.e., each sensor) individually. Afterwards, the tracking results of different data sources are fused to determine which tracks belong to the same object, through the track-to-track association step. Finally, each object's multiple tracks are fused to generate a more accurate detection result.

\subsection{World model layer}

The world model layer aims to process the fusion results of the perception layer and provide the necessary information for the low-level control layer. The fusion results of each detected object are stored to generate a history state (position, heading, speed, acceleration, and wheel angle) of the corresponding object. Based on the state information of the platoon (identities and position order of cooperative vehicles within the platoon), each vehicle identifies the data of the leading vehicle of the platoon and the data of its front vehicle. Such data, which can help generate a world model surrounding the host vehicle, are sent to the low-level control layer to output vehicle control signals. Clearly, to the first following vehicle, the leading vehicle of the platoon is also its front vehicle.

\subsection{Low-level control layer}

The aim of this layer is to calculate the low-level control signal using the output of the world model layer. The main purpose of the manoeuvring operations in CACC is to autonomously control each following vehicle to closely and safely follow its front vehicle. Hence in our system, this layer executes a control algorithm that intends to allow each host vehicle to act longitudinally (through acceleration) based on the trajectory of the front vehicle, and to adjust lateral actions (through steering wheel angle) based on the trajectory of the leading vehicle. The control algorithm is designed following the MPC framework [31] [32] and is elaborated in Section 5.

\subsection{Output layer}

The output data of the five-layer software architecture consist of control action signals used for manoeuvring the host vehicle (sent to the gateway), V2V communication messages to be broadcasted to other vehicles (sent to the LTE$\mathrm{V}$ transceiver), and display messages for the driver (sent to the HMI). The contents of these data are displayed in Table 3.

Table 3 Output layer data

\begin{tabular}{llll}
\hline $\begin{array}{l}\text { Data } \\
\text { receiver }\end{array}$ & $\begin{array}{l}\text { Collection } \\
\text { frequency }\end{array}$ & $\begin{array}{c}\text { Data } \\
\text { description }\end{array}$ & \multicolumn{1}{c}{ Data contents } \\
\hline Gateway & $10 \mathrm{~Hz}$ & $\begin{array}{l}\text { Control } \\
\text { messages }\end{array}$ & $\begin{array}{l}\text { Desired acceleration } \\
\left(\mathrm{m} / \mathrm{s}^{2}\right) \\
\end{array}$ \\
& & $\begin{array}{l}\text { Desired steering } \\
\text { angle (degree) }\end{array}$
\end{tabular}

\begin{tabular}{llll}
\hline HMI & $20 \mathrm{~Hz}$ & Vehicle state & $\begin{array}{l}\text { States of all vehicles } \\
\text { in the platoon } \\
\text { Vehicle order and } \\
\text { speed, platoon } \\
\text { formation, etc. }\end{array}$ \\
$\begin{array}{llll}\text { LTE-V } \\
\text { trans- } \\
\text { ceiver }\end{array}$ & $50 \mathrm{~Hz}$ & Vehicle identity \\
& & $\begin{array}{l}\text { Vehicle state } \\
\text { Global positioning } \\
\text { state }\end{array}$ \\
& & $\begin{array}{l}\text { Platoon state } \\
\text { Platoon } \\
\text { broadcast } \\
\text { information }\end{array}$ & Platoon control \\
& &
\end{tabular}

Good perception and control algorithms are of importance in achieving a reliable and stable platoon. In the next two sections, we describe our perception and control methods.

\section{Perception}

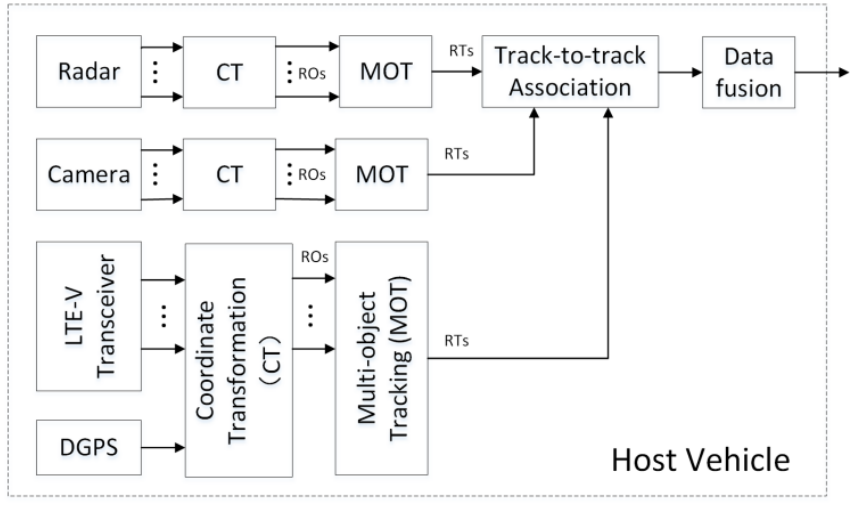

Fig. 5 Perception layer functions.

As discussed in Section 3.1, each vehicle obtains data regarding its surrounding objects, including: (1) states of the other vehicles in the platoon through the LTE-V transceiver, and (2) measurements of nearby objects (vehicles inside or outside the platoon, bicycles, pedestrians, etc.) and obstacles through the radar and camera. (Note that if vehicles outside the platoon can also broadcast their state information using LTE-V, the host vehicle can have the knowledge regarding them from its LTE-V transceiver. But in our platform, this is currently not considered.) The vehicle uses such measurements to construct a representation of the driving environment. The functions conducted in the perception layer are shown in Fig. 5 and are elaborated as follows.

\subsection{Coordinate transformation}

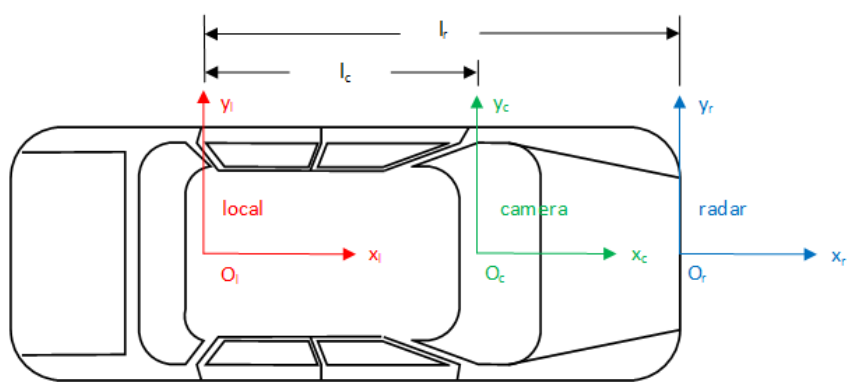

Fig. 6 Vehicle local, radar, and camera coordinate systems.

At each host vehicle, the positioning information received from DGPS receiver (either its own position from 
the DGPS receiver or other vehicles' positions from the LTE$\mathrm{V}$ transceiver) is represented in the global positioning coordinate (spheroidal coordinate) by using WGS84 (World Geodetic System 1984) [36] as the reference coordinate system. The measurements of the locations of nearby objects from radar and camera are represented in their own rectangular coordinate systems. The origin of the radar (resp. camera) coordinate system is set at the installation position. The $\mathrm{x}$-axis and $\mathrm{y}$-axis are set as shown in Fig. 6. In order to establish a unified coordinate system, the locations measured by the DGPS receiver, camera, and radar are all transformed to coordinates in a local rectangular coordinate system whose origin is placed at the centre of the host vehicle's rear axle, as shown in Fig. 6. We use $l_{c}$ (resp. $l_{r}$ ) to denote the distance between the camera (resp. radar) and the rear axle.

For the positioning information obtained from DGPS receiver, we first convert it from the global positioning coordinate system to the UTM coordinate system, and then to the local rectangular coordinate system. For example, assume that we attain the positions of the host vehicle (from its own DGPS receiver) and another vehicle within the platoon (from the LTE-V receiver) in the UTM coordinate system as $\overrightarrow{x_{u_{h}}}$ and $\overrightarrow{x_{u_{o}}}$, respectively. Then after coordinate transformation, the positions in the host vehicle's local rectangular coordinate system respectively become $\overrightarrow{x_{h}}=\overrightarrow{0}$ and

$$
\overrightarrow{x_{l_{o}}}=\left[\begin{array}{cc}
\sin \left(\alpha_{h}\right) & \cos \left(\alpha_{h}\right) \\
-\cos \left(\alpha_{h}\right) & \sin \left(\alpha_{h}\right)
\end{array}\right]\left(\overrightarrow{x_{u_{o}}}-\overrightarrow{x_{u_{h}}}\right)
$$

where $\alpha_{h}$ is the heading of the host vehicle.

In addition, assume that we attain the positions of a nearby object from the camera and the radar in their own coordinate systems as $\overrightarrow{x_{c_{o}}}$ and $\overrightarrow{x_{r_{o}}}$, respectively. Transforming them to the host vehicle's local rectangular coordinate system leads to new positions $\overrightarrow{x_{l_{c}}}$ and $\overrightarrow{x_{l_{r}}}$ respectively:

$$
\begin{aligned}
& \overrightarrow{x_{l_{c}}}=\overrightarrow{x_{c_{o}}}+\left(l_{c}, 0\right)^{T} \\
& \overrightarrow{x_{l_{r}}}=\overrightarrow{x_{u_{o}}}+\left(l_{r}, 0\right)^{T}
\end{aligned}
$$

\subsection{Multi-object tracking}

At each iteration of the perception process, every sensing data source (camera, radar, and LTE-V transceiver) provides the host vehicle with new measurements of the positions of surrounding objects. The multi-object tracking step, carried out using each type of sensing data individually, aims to match the new measurements to the objects that were already tracked in the past iteration. The whole procedure usually consists of three sub-steps: prediction of new locations using previous measurements, association of the previously tracked objects with new measurements, and filtering of the tracking measurements.

[37] proposes a constant velocity (CV) multi-object tracking algorithm that takes both tracking accuracy and realtime performance into consideration. However, since a linear prediction model is applied to predict the positions of moving objects, it may cause relatively large prediction errors in road curves, especially when object's speed is high. We hence consider a hybrid prediction model to address this issue, by combining the CV method and a CTRV (Constant Turn Rate and Velocity) prediction method [38]. The model takes advantage of the V2V communication. For each tracked object (after data fusion of the past iteration), we intend to extract its turn rate from the DGPS state information received through the LTE-V transceiver. If the turn rate is not zero, the CTVR prediction method is applied to attain a better position prediction for non-liner motion. Otherwise, the $\mathrm{CV}$ prediction method with liner prediction is adopted. On the other hand, if DGPS state information is not available, due to unsuccessful V2V transmission or lack of LTE-V transceiver at the object, we cannot find the turn rate. In this case, the CV prediction model is applied. The working procedure of such a hybrid prediction method is illustrated in Fig. 7.

After the predictions of the new locations of the detected objects are obtained, we employ the Mahalanobis distance to determine the degree of association between new measurements and the previously tracked objects (a smaller Mahalanobis distance between a predicted location and a newly measured location indicates that the new measurement and the tracked object are more likely to be associated). Inspired by the method presented in [37], the Hungarian algorithm is used to obtain the best match between measurements and objects. The object association process, operated to each data source individually, is described as follows.

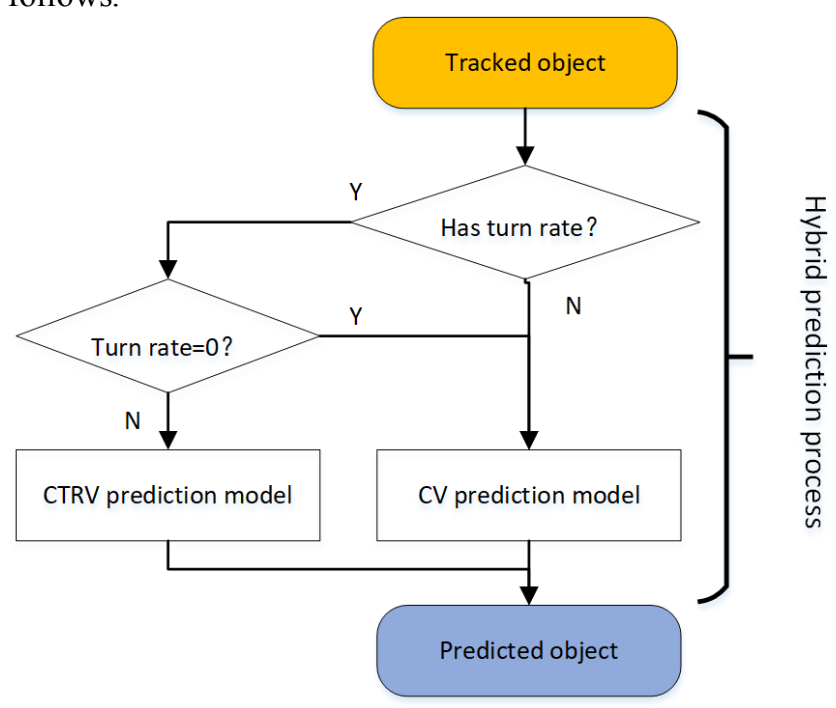

Fig. 7 Hybrid prediction process

We use $\mathcal{D}=\left\{R O_{1}, R O_{2}, \cdots, R O_{N}\right\}$ to denote the set of new measurements from one of the data sources (radar, camera, or LTE-V transceiver) in which $R O_{i}$ denotes the measurement of the $i$ th detected object. We also use $\mathcal{R}=$ $\left\{R O_{1}^{\prime}, R O_{2}^{\prime}, \cdots, R O_{K}^{\prime}\right\}$ to denote the set of predictions of the previously tracked objects. $R O_{i}$ and $R O_{j}^{\prime}$ have the same data form, representing the position and velocity of an object. The Mahalanobis distance between them can be calculated as

$$
S_{i, j}=\left(\overrightarrow{x_{\imath}}-\overrightarrow{x_{J}}\right)^{T} B^{-1}\left(\overrightarrow{x_{\imath}}-\overrightarrow{x_{J}}\right)
$$

in which $\vec{x}_{\imath}$ and $\overrightarrow{x_{j}}$ denote the state vector (position and velocity) of $R O_{i}$ and $R O_{j}^{\prime}$ respectively, and $B$ is the covariance matrix of the object state. By this means, we obtain a matrix form of the Mahalanobis distances for all predictions and new measurements: 


$$
M_{N \times K}=\left[\begin{array}{ccccc}
S_{1,1} & \cdots & S_{1, j} & \cdots & S_{1, K} \\
\vdots & \ddots & \vdots & & \vdots \\
S_{i, 1} & \cdots & S_{i, j} & \cdots & S_{i, K} \\
\vdots & & \vdots & \ddots & \vdots \\
S_{N, 1} & \cdots & S_{N, j} & \cdots & S_{N, K}
\end{array}\right]
$$

The Hungarian algorithm [39], which is a combinatorial optimization algorithm, is applied to solve the object association problem according to the nearest Mahalanobis-distance strategy. It finds the maximum number of matches and the sum of Mahalanobis distance of these matches is the minimum. It is possible that a new measurement is unable to associate with any existing tracked object if the Mahalanobis distance between them is higher than a predefined distance threshold, one example of which can be 9.4877 [37]. Therefore, the obtained number of matches must be less than or equal to $\min \{N, K\}$.

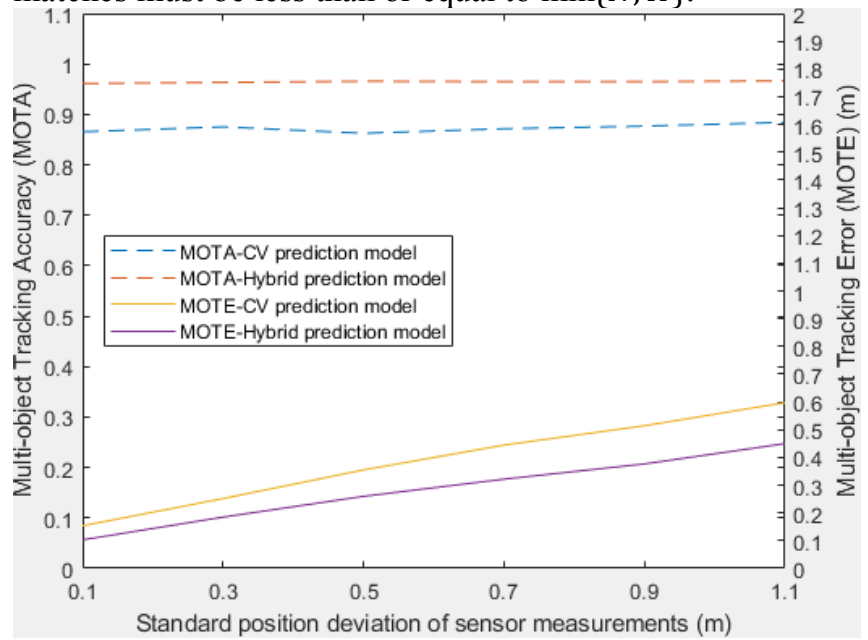

Fig. 8 Comparison of multi-object tracking performance on a road curve.

The matching result at each iteration may lead to two conditions. If a new measurement matches a previously tracked object, it is used to update the state of the tracked object. Otherwise, we generate a new tracked object for the measurement with a new ID. For each object, the list of its state (position and velocity) at all previous iterations (i.e., time instants) is termed the track of the object. Clearly, each data source (radar, camera and LTE-V transceiver) provides a set of such tracks, each of which represents one object.

In order to test the effectiveness of our hybrid prediction model, we use PreScan to conduct a simulation with a platoon of two vehicles driving with the speed of $15 \mathrm{~m} / \mathrm{s}$ on a road curve. Fig. 8 shows the performance comparison of multi-object tracking with hybrid prediction and the conventional $\mathrm{CV}$ prediction model. Our method can have better performance in terms of both MOTA (multi-object tracking accuracy) and MOTE (multi-object tracking error) [40].

\subsection{Track-to-track association}

So far, at every iteration of the perception process, each of the three sensing data sources, i.e., radar, camera, and LTE-V transceiver, provides the host vehicle with a set of tracks to represent the status of nearby objects (cooperative vehicles within the platoon, and other vehicles and objects outside the platoon). The track-to-track association step in the perception layer intends to identify the tracks from different data sources that are associated with the same object. Based on the minimum distance and Chi-square test, [41] proposes a multi-sensor multi-target track association (MTA) technique to support such a function for two sensing data sources. In our system, we further extend the method to potentially support three data sources.

Assume that at time instant (iteration) $k$, the three sensing data sources have a total of $M_{A}$ tracks. Use $\mathcal{T}=$ $\left\{R T_{1}, \cdots, R T_{M_{A}}\right\}$ to denote the set which contains all these tracks. Note that $M_{A}$ can be different in different time instants. Considering the fact that any two tracks $R T_{i}$ and $R T_{j}$ in $\mathcal{T}$ may have different sizes, we use $M_{i j}$ to denote the smaller track size of $R T_{i}$ and $R T_{j}$. The average (time average, over $M_{i j}$ historical measurements) distance between them is calculated as

$$
D^{k}\left(R T_{i}, R T_{j}\right)=\frac{1}{M_{i j}} \sum_{i=0}^{M_{i j}-1} d^{k-i}\left(R T_{i}, R T_{j}\right)
$$

with

$$
d^{t}\left(R T_{i}, R T_{j}\right)=\sqrt{\left(\overrightarrow{x_{l}^{t}}-\overrightarrow{x_{J}^{t}}\right)^{T}\left(\overrightarrow{x_{l}^{t}}-\overrightarrow{x_{J}^{t}}\right)}
$$

where $\overrightarrow{x_{l}^{t}}$ and $\overrightarrow{x_{j}^{t}}$ respectively denote the positions of $R T_{i}$ and $R T_{j}$ at time instant $t\left(\mathrm{t} \in\left\{k-M_{i j}+1, \cdots, k\right\}\right)$.

A smaller average distance between two tracks implies that with higher probability the two tracks represent the same object. In order to identify the (possibly more than two) tracks in $\mathcal{T}$ that represent each single object, similar to [38], for each pair of tracks $R T_{i}$ and $R T_{j}$ we define

$$
R_{i, j}^{k}=\left\{\begin{array}{ll}
1, & \text { if } D^{k}\left(R T_{i}, R T_{j}\right) \leq \alpha \\
0, & \text { if } D^{k}\left(R T_{i}, R T_{j}\right)>\alpha
\end{array},\right.
$$

where $\alpha$ is a distance threshold determined in experiments. By this means, an adjacency matrix can be obtained as:

$$
A_{M_{A} \times M_{A}}=\left[\begin{array}{ccc}
R_{1,1}^{k} & \cdots & R_{1, M_{A}}^{k} \\
\vdots & \ddots & \vdots \\
R_{M_{A}, 1}^{k} & \cdots & R_{M_{A}, M_{A}}^{k}
\end{array}\right]
$$

$A_{M_{A} \times M_{A}}$ represents the relationship among all the $R T \mathrm{~s}$ in $\mathcal{T}$. Applying the community detection algorithm [42] outputs a set of track-clusters, each of which contains tracks belonging to the same object.

\subsection{Data fusion}

The last step of the perception layer is to fuse the tracks in each track-cluster obtained in the track-to-track association step to determine the position of the detected object. The data fusion strategies are different due to the availability of data tracks from different sensing data sources.

First, consider a track-cluster that contains the track generated by data collected from the LTE-V transceiver (i.e., the representing object is another vehicle in the platoon). As described in Section 2, in our system each vehicle measures its own position by DGPS, and then sends the position information to other cooperative vehicles within the platoon. The accuracy is within $0.01 \mathrm{~m}$ and is in general much higher than the measurement results of radar and camera. Therefore, 
in this case, the data fusion step directly chooses the track of the LTE-V transceiver and ignores the other two.

Albeit that such a data track provides sufficiently high positioning accuracy, receiving it via $\mathrm{V} 2 \mathrm{~V}$ communication may face two potential problems. First, the transmitter vehicle may not be able to receive DGPS signals under certain driving conditions, such as driving in a tunnel, under a bridge, or in an indoor parking lot. In addition, although LTE-V has the potential to provide highly-reliable and low-delay transmission, wireless communication in a highly mobile environment may still be an uncertain event, due to the impact of random fading, interference, and noise. Hence to guarantee the platoon to operate safely and efficiently, each following vehicle must be able to deal with the situation when LTE-V signals are (temporarily) unavailable. Furthermore, other vehicles and objects outside the platoon may not be capable of cooperating with the platoon, due to insufficient coordination mechanism, unwillingness to cooperate, or lack of DGPS/LTE-V devices. For all these cases, a track-cluster obtained from the track-to-track association step may contain tracks generated by only the radar and camera. Note that the front-facing radar in our testbed provides more accurate measurements in the longitudinal direction whereas the camera provides more accurate measurements in the lateral direction. Therefore, the object's longitudinal measurements from radar and lateral measurements from camera at the same time instants are combined to obtain a new track for further processing.

Finally, in situations where an object is detected only by either the camera or radar, the associated track-cluster has one element. This track is naturally the single source for measuring the location of the object.

In order to make a better use of the track attained in one of the above three conditions to locate the detected object, we apply a linear Kalman filter (KL) to combine the measurement of the current iteration and those of the previous iterations to calculate a smoother and more precise position representation of the object. The results of all surrounding objects are output to the world-model layer for generating a precise world model of the platoon, in order to support decision making for low-level control.

\section{Low-level Control}

The low-level control layer operates control algorithms that determine the desired acceleration used for longitudinal control and the desired steering angle used for lateral control. In this section, we first discuss the control algorithm used for longitudinal control, followed by that for lateral control. The control algorithms presented in the subsections are based on the MPC framework [31] [32] and distributed MPC schemes [43] [44]. This means every following vehicle in the platoon solves its own MPC problem based on local information. The main advantage of using MPC is that it has straightforward formulation and explicit handling of constraints with fast processing capability.

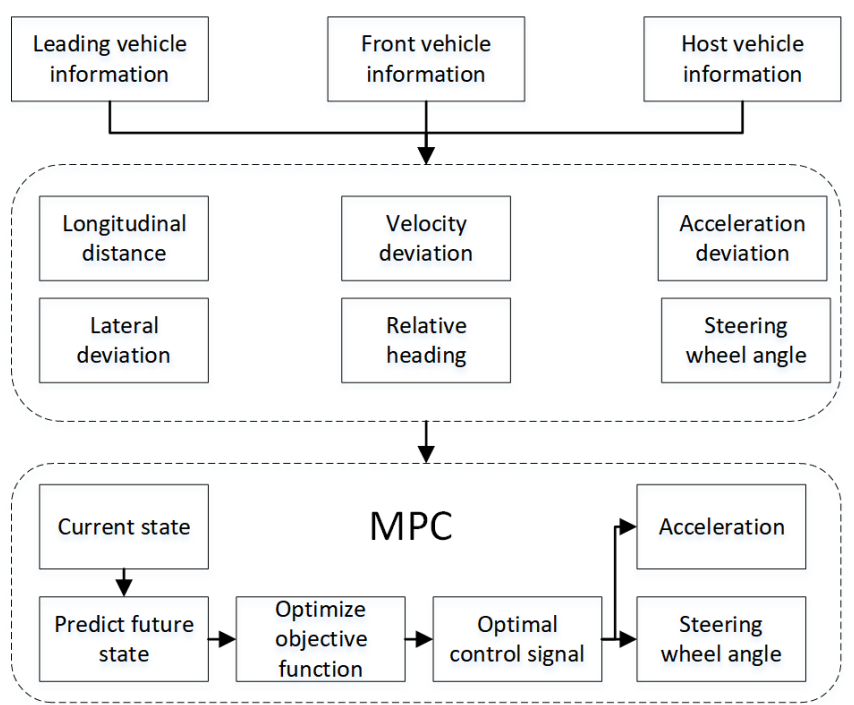

Fig. 9 Data flow and working process of the low-level control layer

\subsection{Longitudinal control}

To realize CACC, the objectives of the longitudinal control are to keep the same speed as and a desired distance to the front vehicle while taking comfort into consideration (appropriate acceleration). To accomplish these objectives, a vector of the deviations of three groups of important variables are defined as the system state:

$$
x=\left[\begin{array}{c}
h^{*}-h \\
\Delta v \\
\Delta a
\end{array}\right]
$$

where $h^{*}$ and $h$ denote the desired distance and the actual distance between the host vehicle and its front vehicle, respectively, $\Delta v$ and $\Delta a$ denote the relative speed and the relative acceleration between the host vehicle and its front vehicle, respectively. The aim of the longitudinal controller is to regulate $x$ in (10) towards the state of $[0,0,0]^{T}$.

With the definition of system state, the state dynamics for longitudinal control is defined as:

$$
x(k+1)=A_{k} x(k)+B_{k} u(k)+C_{k}
$$

where $u(k)$ denotes the control variable of acceleration at prediction time $k$ and

$$
\begin{gathered}
A_{k}=I_{3}+\left[\begin{array}{ccc}
0 & -1 & 0 \\
0 & 0 & 1 \\
0 & 0 & -1 / \tau_{a}
\end{array}\right] \times t_{r} \\
B_{k}=\left[\begin{array}{c}
0 \\
0 \\
-1 / \tau_{a}
\end{array}\right] \times t_{r} \\
C_{k}=\left[\begin{array}{c}
0 \\
0 \\
a_{l} / \tau_{a}
\end{array}\right] \times t_{r}
\end{gathered}
$$

In (12)-(14), $\tau_{a}$ denotes the first order inertia delay of the throttle/brake system of the host vehicle, $a_{l}$ is the acceleration of the leading vehicle in the platoon, and $t_{r}$ is a small step size for model predictive control. 
The cost function used in the longitudinal controller is:

$$
J=\int_{t=0}^{T_{p}}\left(\frac{1}{2} x^{\prime} Q x+\frac{1}{2} u^{\prime} R u\right) d t
$$

where $x$ is the system state and $u$ is the control input. $T_{p}$ is the value of the prediction horizon. In our system the temporal MPC algorithm is used to keep a stable distance to the front vehicle at every predictive time and we set $t_{r}=0.1 \mathrm{~s}$, $T_{p}=10 \mathrm{~s} . Q$ and $R$ are, respectively, the weight matrices of the system state and control variable defined as:

$$
Q_{k}=\left[\begin{array}{ccc}
\beta_{1} & 0 & 0 \\
0 & \beta_{2} & 0 \\
0 & 0 & \beta_{3}
\end{array}\right], R_{k}=\beta_{4}
$$

where, $\beta_{1}, \beta_{2}, \beta_{3}$ and $\beta_{4}$ are the parameters adjusted and determined through experiments.

\subsection{Lateral control}

The objective of the lateral controller is to keep tracking the trajectory of the leading vehicle with the desired lateral deviation of 0 . To accomplish this mission, we define the state vector as:

$$
x=\left[\begin{array}{c}
\Delta f \\
\varphi_{f}-\varphi \\
\delta
\end{array}\right]
$$

where $\Delta f$ is the lateral deviation between the host vehicle and the history trajectory of the leading vehicle. We select the position in the history trajectory of the leading vehicle which is the closest to the host vehicle. Then $\varphi_{f}$ denotes the heading of the leading vehicle at this chosen position. $\varphi, \delta$ are the heading and the steering angle of the host vehicle, respectively. The aim of the lateral controller is to regulate $x$ towards the state of $[0,0,0]^{T}$.

The state dynamics for lateral control is similar to (11). But unlike the definition in longitudinal control which uses the current state of the front vehicle as a reference, the history path of the leading vehicle is used. Furthermore, the control input $u$ denotes the steering angle of the host vehicle at prediction time $k$ and $A_{k}, B_{k}$ and $C_{k}$ are defined as:

$$
\begin{gathered}
A_{k}=I_{3}+\left[\begin{array}{ccc}
0 & -1 & 0 \\
0 & 0 & -1 / L \\
0 & 0 & -1 / \tau_{\delta} v
\end{array}\right] \times x_{r} \\
B_{k}=\left[\begin{array}{c}
0 \\
0 \\
1 /\left(\tau_{\delta} v\right)
\end{array}\right] \times x_{r} \\
C_{k}=\left[\begin{array}{c}
-\varphi_{f} \\
k \\
0
\end{array}\right] \times x_{r}
\end{gathered}
$$

where, $\tau_{\delta}$ denotes the first order inertia delay of the steering system and $L$ is the length of the axle of the host vehicle. $k$ is the curvature of the history trajectory of the leading vehicle and $x_{r}$ denotes a small step size for model predictive control.

The cost function is defined as:

$$
J=\int_{s=0}^{S_{p}}\left(\frac{1}{2} x^{\prime} Q x+\frac{1}{2} u^{\prime} R u\right) d s
$$

where $x$ is the system state and $u$ is the control input. $S_{p}$ is the value of the prediction horizon. The spatial domain (instead of temporal domain) MPC is used for lateral control with control step $x_{r}=0.1 \mathrm{~m}, S_{p}=10 \mathrm{~m}$ which means the preview path with 100 points will be calculated for lateral control. $Q$ and $R$ are the weight matrices of the system state and control variable, respectively:

$$
Q_{k}=\left[\begin{array}{ccc}
\beta_{5} & 0 & 0 \\
0 & \beta_{6} & 0 \\
0 & 0 & \beta_{7}
\end{array}\right], R_{k}=\beta_{8}
$$

where $\beta_{5}, \beta_{6}, \beta_{7}$ and $\beta_{8}$ are the parameters determined during the experiments.

The aims of both longitudinal and lateral controllers are to minimize the cost function to attain the optimal control input $u$. The Newton methods [45] are used to solve the nonlinear optimization problems. At the end of each calculation, the optimal acceleration and steering angle of the host vehicle are sent to the actuators for automated driving. The calculation is executed at the frequency of $10 \mathrm{~Hz}$. The details of the data flow and the operation process are shown in Fig. 9.

\section{Field Experiments and Analyses}

We have integrated the hardware and software architectures described in the above sections into a complete testbed platform for supporting CACC with two or three SAIC Morris Garages cars. A number of field experiments were conducted, in the National Intelligent Connected Vehicles (Shanghai) Pilot Zone of China, Shanghai, China, to verify the feasibility and performance of the platform. In what follows, we present some of our experiment results.

\subsection{Performance of perception layer}

We first demonstrated the functions of the perception layer. In the experiments, two cars formed a platoon with inter-vehicle gap of $10 \mathrm{~m}$. The leading vehicle was driven by a human driver, and the following vehicle was manoeuvred by our cooperative automated driving system. Two different driving scenarios were taken into consideration. The first was a road curve, on which the platoon drove at the speed of $20 \mathrm{~km} / \mathrm{h}$. The second was a straight road, on which the platoon's speed was around $60 \mathrm{~km} / \mathrm{h}$.

In our experiments, we did not consider certain extreme driving conditions where the DGPS or LTE-V connections were lost. Therefore, the following vehicle always attained accurate position information (within $0.01 \mathrm{~m}$ ) of the leading vehicle through the V2V communication. To verify the function of the perception layer, we first separated the data of the LTE-V transceiver from the layer input. This means that, as conventional ACC solutions, the perception layer of the following vehicle used only the measurements of radar and camera to establish its estimation of the leading vehicle's trajectory. The accurate trajectory that the leading vehicle obtained from the data of the LTE-V transceiver was treated as a baseline ground truth for evaluating the performance of the camera and radar measurements.

Three different trajectory estimation scenarios were considered: the following vehicle attained the driving trajectory of the leading vehicle through object tracking and 
association respectively using (1) the tracks of the radar, (2) the tracks of the camera, and (3) fusion of the tracks of radar and camera. The performance of the former two scenarios can demonstrates the feasibility of the multi-object tracking step of our system. And that of the last scenario shows the feasibility of track-to-track association and data fusion steps in the perception layer.

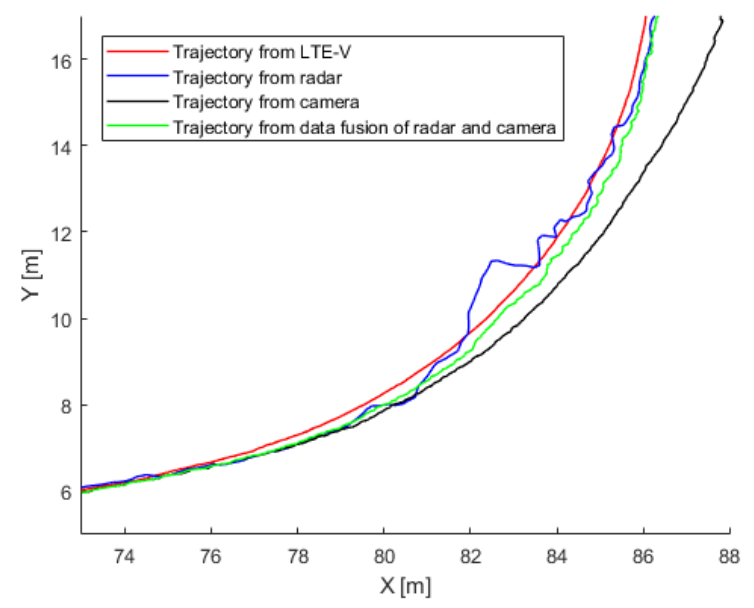

Fig. 10 Experimental results on a road curve.

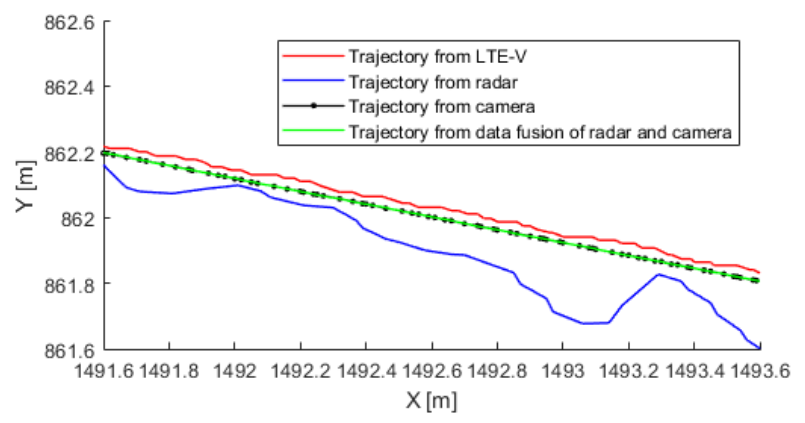

Fig. 11 Experimental results on a straight road.

Table 4 Error statistics on road curve/straight road

\begin{tabular}{lll}
\hline & \multicolumn{1}{c}{ Average $(\mathrm{m})$} & \multicolumn{1}{c}{ Variance $\left(\mathrm{m}^{2}\right)$} \\
\hline Radar & $0.66 / 0.31$ & $0.08 / 0.02$ \\
Camera & $0.60 / 0.72$ & $0.32 / 0.04$ \\
Fusion & $0.45 / 0.24$ & $0.04 / 0.01$
\end{tabular}

Fig. 10 displays one experiment result on the road curve. It can be seen that as the platoon drove along the road curve, the estimation of the leading vehicle's trajectory using camera alone gradually deviated from the true trajectory (attained from V2V transmission of DGPS signals). The estimation using radar alone was relatively close to the ground truth, but with variations due to measurement errors and difficulties in object association. Fusing the tracks obtained from both radar and camera led to a smoother and more accurate trajectory estimation. (The average and variance of the estimation errors in the experiment are displayed in Table 4. Note that statistics were obtained using both longitudinal direction errors and lateral direction errors. Only the latter can be visualized in Fig. 10.). However, the distance to the true trajectory is also notable. As a result, providing automated driving with collaboration capability through the LTE-V technology has the potential to significantly improve the environment perception quality of each individual vehicle.

Fig. 11 shows part of results on the straight road. In this case, the trajectory attained from camera alone was closer to the true trajectory of the leading vehicle, compared with that from radar alone. The difference between the trajectory from data fusion and that from LTE-V transceiver (i.e., ground truth) is still observable. In practice, the true driving environment would contain the combinations of both road scenarios. CACC should be established upon sufficiently accurate measurements of cooperative vehicles within the platoon. This calls for the necessity of high-quality vehicular communications.

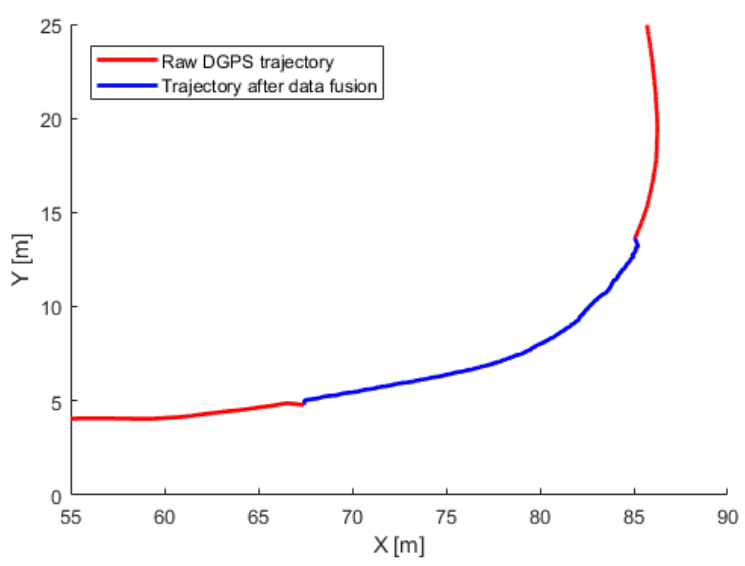

Fig. 12 Experimental result when $\mathrm{V} 2 \mathrm{~V}$ signals were lost.

As mentioned in Section 4, the data fusion output step of the perception layer provides the mechanism to deal with potential failure of $\mathrm{V} 2 \mathrm{~V}$ communication (DGPS or LTE-V signals are lost due to, e.g., harsh driving environment). To demonstrate this function, we demanded the perception layer to use only the data fusion result of the camera and radar to estimate the trajectory of the leading vehicle for some time, in an experiment on a road curve. Hence, when the following vehicle received signals from $\mathrm{V} 2 \mathrm{~V}$ communication, it utilized the data track of the LTE-V transceiver. Otherwise, it switched its data fusion input to the tracks of the camera and radar. The result is shown in Fig. 12. It can be seen that the trajectory estimations were not significantly affected near the switching positions. This enables the functions in the lowlevel control layer to be operated smoothly even when V2V communication and vehicle cooperation are temporarily unavailable.

\subsection{Performance of low-level control layer}

In this subsection, we present the experiment results for evaluation of the functions of the low-level control layer. In the first experiment, three vehicles form a platoon driving on a straight road. The leading vehicle, driven by a human driver, started from a still position and steadily accelerated. After the speed reached around $34 \mathrm{~km} / \mathrm{h}(9.5 \mathrm{~m} / \mathrm{s})$, it rapidly decelerated until it completely stopped. Its speed profile in the experiment is shown in Fig. 13 (as Vehicle No. 1). The other two autonomously driven vehicles intended to follow the leading vehicle as a platoon with inter-vehicle gap of $10 \mathrm{~m}$. Their speed profiles are also displayed in Fig. 13 as Vehicle 
No. 2 (the first following vehicle) and Vehicle No. 3 (the second following vehicle).

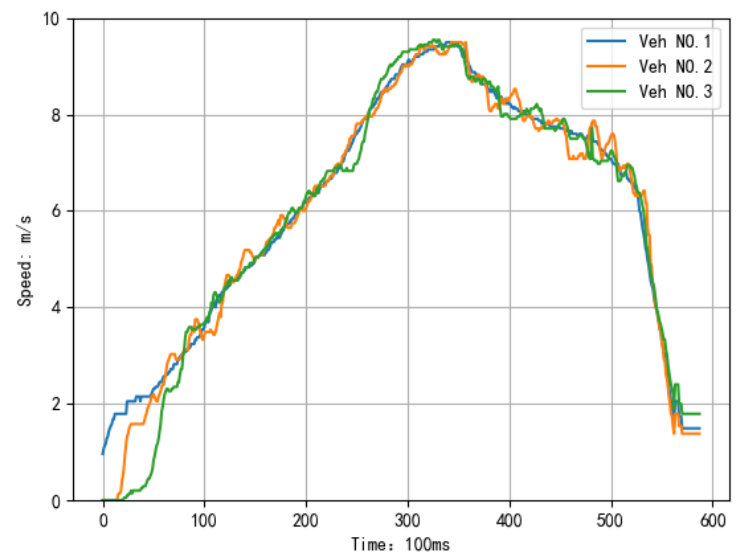

Fig. 13 Experimental results of the speed of the platoon.

From the figure we can see that the two following vehicles rapidly responded to the movement of the leading vehicle. They automatically adapted their speed to almost the same level as the leading vehicle, to keep a constant intervehicle distance. Compared with the second vehicle, the third vehicle's speed variation was larger and lagged behind, because it planned its longitudinal control actions according to the movements of the second vehicle, i.e., its front vehicle. Due to LTE-V technology, the third vehicle obtained the states of its front vehicle with very small delay. The speed variation was acceptable and did not cause comfortlessness.

Fig. 14 displays the difference between the desired inter-vehicle distance and the true inter-vehicle distance in the experiment, for the two following vehicles, in both longitudinal and lateral directions. The results reflected the low-layer control performance of our system. It is seen that most of the longitudinal differences were within a $\pm 25 \mathrm{~cm}$ region (marked by dash lines), and most of the lateral differences were within the $\pm 2 \mathrm{~cm}$ region. The third vehicle had delayed and larger distance deviation than the second vehicle, since it had to adjust its actions based on the behaviour of its front vehicle. But since the differences for both vehicles were small, the functions of the both longitudinal and lateral control algorithms of our system presented in Section 5 were demonstrated.
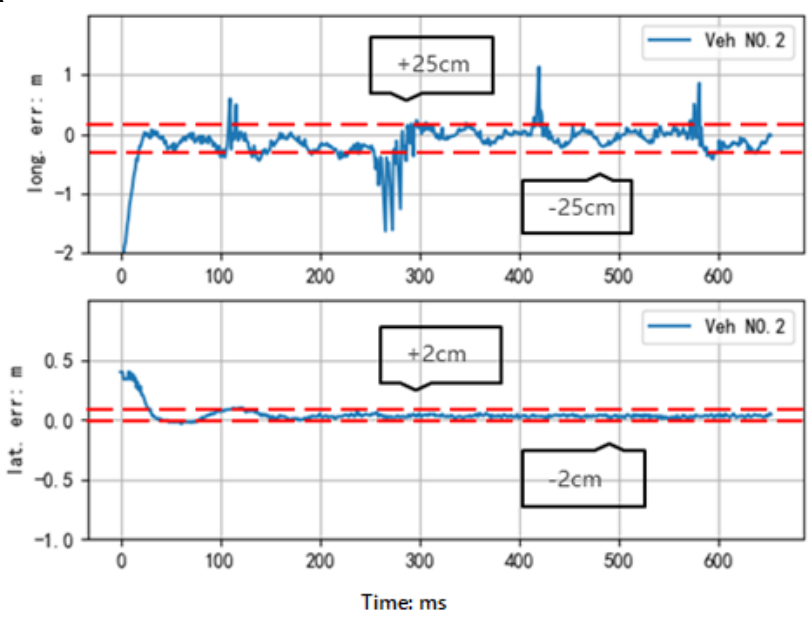

(a) Vehicle No. 2
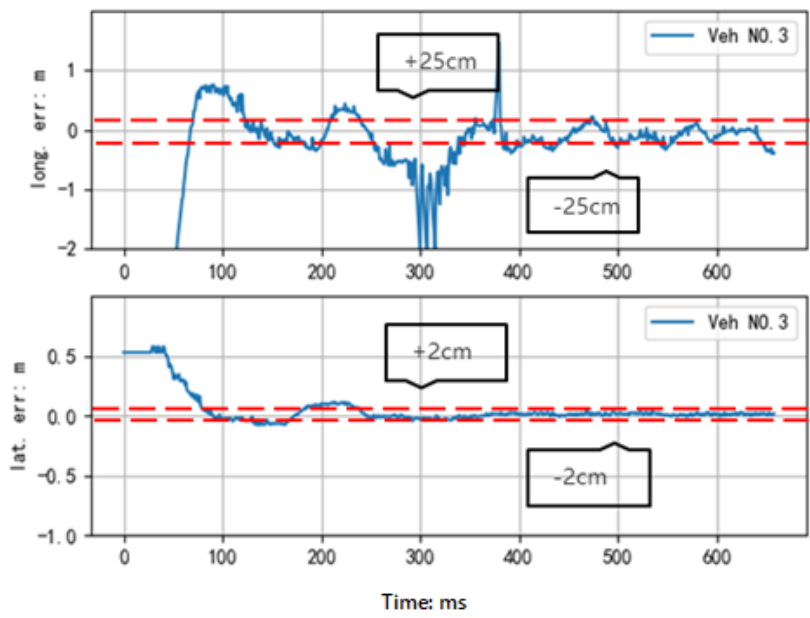

(b) Vehicle No. 3

Fig. 14 Experimental results of longitude and latitude distance deviations

Table 5 Low-level control performance

\begin{tabular}{lcccl}
\hline scenarios & $\begin{array}{c}\text { velocity } \\
(\mathrm{km} / \mathrm{h})\end{array}$ & $\begin{array}{c}\text { turning } \\
\text { radius } \\
(\mathrm{m})\end{array}$ & $\begin{array}{c}\text { maximal } \\
\text { longitudinal } \\
\text { error }(\mathrm{cm})\end{array}$ & $\begin{array}{c}\text { maximal } \\
\text { lateral } \\
\text { error }(\mathrm{cm})\end{array}$ \\
\hline $\begin{array}{l}\text { Lane } \\
\text { changing }\end{array}$ & $0-30$ & $>250$ & 35 & 20 \\
$\begin{array}{l}\text { Turning at } \\
\text { right-angled }\end{array}$ & $0-30$ & $>25$ & 30 & 20 \\
$\begin{array}{l}\text { bend } \\
\text { Circling }\end{array}$ & $0-20$ & $>15$ & 35 & 20 \\
$\begin{array}{l}\text { Turning } \\
\text { around }\end{array}$ & $0-20$ & $>5$ & 40 & 20 \\
$\begin{array}{l}\text { S-shaped } \\
\text { curvilinear } \\
\text { movement }\end{array}$ & $0-30$ & $>18$ & 30 & 15
\end{tabular}

We also carried out several experiments with a platoon of two vehicles when the leading vehicle performed actions including lane changing, turning at right-angled bend, circling, turning around, and s-shaped curvilinear movement. In all experiments, the following vehicle intended to track the trajectory of the leading vehicle and keep a fixed distance of $10 \mathrm{~m}$. The descriptions of the leading vehicle in different actions (in terms of velocity and turning radius) and the error performance (rounded to multiple of $5 \mathrm{~cm}$ ) in the experiments are presented in Table 5. The observation that the differences between desired and actual inter-vehicle distances were relatively small verified the feasibility of our CACC testbed platform.

\section{Conclusion}

Under the concept of CACC, forming vehicles on the road into compact platoons is potentially an effective approach to addressing the problems occurred in today's road transportation systems. Platooning with intelligent vehicles can release the burden of human drivers, avoid road accidents, improve traffic efficiency, and reduce fuel consumption and environment pollution. We have developed a testbed platform for realizing CACC where a number of automated vehicles closely follow a human-driven leading vehicle through cooperation enabled by the LTE-V technology. The platform is developed on vehicles equipped with a suite of on-board sensing, communication, and computing devices. Supported 
by a five-layer software architecture, accurate perception of the state of the platoon and intelligent control of the vehicles are realized. The functions of our testbed platform have been verified by several initial field experiments. Further system optimization and experiments in more complicated and practical driving environments will be conducted as future works.

\section{Acknowledgment}

This work was partially supported by National Natural Science Foundation of China (Grant No. 61803284), Fundamental Research Funds for the Central Universities (No. 1600219316), and Shanghai Yangfan Program (No. 18YF1424200). C. Wang's work was a part of a project that has received funding from the European Union's Horizon 2020 research and innovation programme under the Marie Sklodowska-Curie grant agreement No 752979. This work reflects only the authors' view and the EU Commission is not responsible for any use that may be made of the information it contains.

\section{Reference}

[1] Xu Z., Zhang K., Min H., et al.: 'What Drives People to Accept Autonomous Vehicles? Findings from A Field Experiment', Transportation Research-Part C: Emerging Technologies, 2018, 95, (10), pp. 320-334

[2] Yamazaki H., Okamoto K., Aoki K.: 'Improvement of Traffic Flow and Fuel Saving Effect by the Trucks of Single File Platooning,, JSAE Annual Congress (Fall), 2010, 64, pp. 61-66

[3] Arem B., Van Driel C., Visser R.: 'The Impact of Cooperative Adaptive Cruise Control on Traffic-flow Characteristics', IEEE Transactions on Intelligent Transportation Systems, 2006, 7, (4), pp. 429-436

[4] Xie D., Zhao X., He Z.: 'Heterogeneous Traffic Mixing Regular and Connected Vehicles: Modeling and Stabilization', IEEE Transactions on Intelligent Transportation Systems, 2018, 13, pp. 1-12

[5] He Z., Zheng L., Song L., et al.: 'A Jam-Absorption Driving Strategy for Mitigating Traffic Oscillations', IEEE Transactions on Intelligent Transportation Systems, 2016, 18, (4), pp. 802-813

[6] He Z., Zheng L., Lu L., et al.: 'Erasing Lane Changes from Roads: A Design of Future Road Intersections', IEEE Transactions on Intelligent Vehicles, 2018, 3, (2), pp. 173184

[7] He Z., Zheng L., Chen P., et al.: 'Mapping to Cells: A Simple Method to Extract Traffic Dynamics from Probe Vehicle Data', Computer-Aided Civil and Infrastructure Engineering, 2017, 32, (3), pp. 252-267

[8] 'Fuel Saving Achieved in the Field Test of Two Tandem

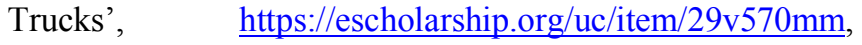
accessed November 2018

[9] Bergenhem C., Huang Q., Benmimoun A., et al.: 'Challenges of Platooning on Public Motorways', Proceedings of 17th ITS World Congress, February 2010, pp. 57-62

[10] Ma C., He R.: 'Green Wave Traffic Control System Optimization Based on Adaptive Genetic-artificial Fish Swarm Algorithm', Neural Computing and Applications, 2015, 26, (5), pp.1-11
[11] Lin Q., Li S. E., Du X., et al.: 'Minimize the Fuel Consumption of Connected Vehicles between Two RedSignalized Intersections in Urban Traffic', IEEE Transactions on Vehicular Technology, 2018, 67, (10), pp. 9060-9072

[12] Ma C., Hao W., He R., et al.: 'Distribution Path Robust Optimization of Electric Vehicle With Multiple Distribution Centers', Public Library of Science One, 2018, 13, (3), pp. 116

[13] Tsugawa S.: 'A Survey on Effects of ITS-related Systems and Technologies on Global Warming Prevention', Control in Transportation Systems, 2009, 42, (15), pp. 334341

[14] Bergenhem C., Shladover S., Coelingh E., et al.: 'Overview of Platooning Systems', Proceedings of the 19th ITS World Congress, Vienna, Austria, 2012, pp. 103-108

[15] Rajamani R., Tan H., Law B., et al.: 'Demonstration of Integrated Longitudinal and Lateral Control for the Operation of Automated Vehicles in Platoons', IEEE Transactions on Control Systems Technology, 2000, 8, (4), pp. 695-708

[16] Shladover S., Lu X., Yang S., et al.: 'Cooperative Adaptive Cruise Control (CACC) for Partially Automated Truck Platooning,, UC Berkeley: California Partners for Advanced Transportation Technology, 2018

[17] Tsugawa S.: 'An Overview on an Automated Truck Platoon within the Energy ITS Project', Control in Transportation Systems, 2013, 46, (21), pp. 41-46

[18] Ma C., Hao W., Wang A., et al.: 'Developing a Coordinated Signal Control System for Urban Ring Road under the Vehicle-infrastructure Connected Environment', IEEE Access, 2018, 6, pp. 52471-52478

[19] Zhao W., Xu L., Bai J., et al.: 'Sensor-based Risk Perception Ability Network Design for Drivers in Snow and Ice Environmental Freeway: A Deep Learning and Rough Sets Approach', Soft Computing, 2017, 22, (5), pp. 14571466

[20] Zhao W., Xu L., Xi S., et al.: 'A Sensor-Based Visual Effect Evaluation of Chevron Alignment Signs' Colors on Drivers through the Curves in Snow and Ice Environment', Journal of Sensors, 2017, 3, pp. 1-10

[21] Kang L., Zhao W., Qi B., et al.: 'Augmenting SelfDriving with Remote Control: Challenges and Directions', In Proceedings of the 19th International Workshop on Mobile Computing Systems \& Applications, 2018, pp. 19-24

[22] Qi B., Suman B.: 'GonioSense: A Wearable-based Range of Motion Sensing and Measurement System for Body Joints: Poster', Proceedings of the 22nd Annual International Conference on Mobile Computing and Networking, 2016

[23] Dolk V., Ouden J., Steeghs S., et al.: 'Cooperative Automated Driving for Various Traffic Scenarios: Experimental Validation in the GCDC 2016', IEEE Transactions on Intelligent Transportation Systems, 2017, 19, (4), pp. 1308-1321

[24] Tas O., Salscheider N., Poggenhans F., et al.: 'Making Bertha Cooperate-Team AnnieWAY's Entry to the 2016 Grand Cooperative Driving Challenge', IEEE Transactions on Intelligent Transportation Systems, 2017, 19, (4), pp. 1262-1276

[25] Kenney J.: 'Dedicated Short-Range Communications (DSRC) Standards in the United States', Proceedings of the IEEE, 2011, 99, (7), pp. 1162-1182

[26] SAE Tech., 'Rep. J2735-200911: Dedicated Short Range Communications (DSRC) Message Set Dictionary', November 2009 
[27] Chen S., Hu J., Shi Y., et al.: 'LTE-V: A TD-LTE-Based V2X Solution for Future Vehicular Network', IEEE Internet of Things Journal, 2017, 3, (6), pp. 997-1005

[28] SAE China Tech. Rep., 'T/CSAE53-2017: Cooperative Intelligent Transportation System; Vehicular Communication; Application Layer Specification and Data Exchange Standard', 2017

[29] '3GPP TR 22.886', http://www.3gpp.org, accessed November 2018

[30] Xu Z., Li X., Zhao X., et al.: 'DSRC Versus 4G-LTE for Connected Vehicle Applications: A Study on Field Experiments of Vehicular Communication Performance', Journal of Advanced Transportation, 2017, 2017, pp. 1-10

[31] Wang M., Daamen W., Hoogendoorn S.: 'Rolling Horizon Control Framework for Driver Assistance Systems. Part I: Mathematical Formulation and Non-cooperative Systems', Transportation Research Part C, 2014, 40, (1), pp. 271-289

[32] Wang M., Daamen W., Hoogendoorn S.: 'Rolling Horizon Control Framework for Driver Assistance Systems. Part II: Cooperative Sensing and Cooperative Control', Transportation Research Part C, 2014, 40, (1), pp. 290-311

[33] Ma J., Hu J., Ed L., et al.: 'An Eco-Drive Experiment on Rolling Terrains for Fuel Consumption Optimization with Connected Automated Vehicles', Transportation Research Part C: Emerging Technologies, 2019, 100, (2019), pp. 125141

[34] Cui L., Hu J., Brian P., et al.: 'Development of a Simulation Platform for Safety Impact Analysis Considering Vehicle Dynamics, Sensor Errors, and Communication Latencies: Assessing Cooperative Adaptive Cruise Control under Cyber Attack', Transportation Research Part C: Emerging Technologies, 2018, 97, (2018), pp. 1-22

[35] Behere S., Torngren M., Chen D.: 'A Reference Architecture for Cooperative Driving', Journal of Systems Architecture, 2013, 59, (10), pp. 1095-1112

[36] Smith R.: 'Department of Defense World Geodetic System 1984: Its Definition and Relationships with Local Geodetic Systems', Defense Mapping Agency, 1987

[37] Wojke N., Bewley A., Paulus D.: 'Simple Online and Realtime Tracking with A Deep Association Metric', IEEE International Conference on Image Processing, 2017, pp. 3645-3649

[38] Schubert R., Adam C., Obst M., et al.: 'Empirical Evaluation of Vehicular Models for Ego Motion Estimation', IEEE Intelligent Vehicles Symposium (IV), 2011, pp. 534 539

[39] Munkres J.: 'Algorithms for the Assignment and Transportation Problems', Journal of the Society for Industrial, 1957, 5, (1), pp. 32-38

[40] Bernardin K., Stiefelhagen R.: 'Evaluating Multiple Object Tracking Performance: the CLEAR MOT Metrics', Journal on Image and Video Processing, 2008, 1, pp. 1-10

[41] Sakr A., Bansal G.: 'Cooperative Localization via DSRC and Multi-sensor Multi-target Track Association', IEEE International Conference on Intelligent Transportation Systems, Rio de Janeiro, Brazil, 2016, pp. 66-71

[42] Li W., Schuurmans D.: 'Modular Community Detection in Networks', Proceedings of the International Joint Conference on Artificial Intelligence, Barcelona, Catalonia, Spain, 2011, pp. 1366-1371

[43] Negenborn R. R., M J., Maestre: 'Distributed Model Predictive Control: An Overview and Roadmap of Future
Research Opportunities', IEEE Control Systems, 2014, 34, (4), pp. 87-97

[44] Bai Y., Zhang Y., Hu J.: 'Cooperative Lane Changing Strategies for Connected Automated Vehicles under Model Predictive Control Framework', 14th International Symposium on Advanced Vehicle Control (AVEC), Beijing, 2018

[45] Deuflhard P.: 'Newton Methods for Nonlinear Problems', Springer, 2004, 28, (6), pp. 1299-1316 